\title{
Control of CNS Synapse Development by $\gamma$-Protocadherin-Mediated Astrocyte-Neuron Contact
}

\author{
Andrew M. Garrett and Joshua A. Weiner \\ Department of Biology, Neuroscience Graduate Program, The University of Iowa, Iowa City, Iowa 52242
}

Recent studies indicate that astrocytes, whose processes enwrap synaptic terminals, promote synapse formation both by releasing soluble factors and through contact-dependent mechanisms. Although astrocyte-secreted synaptogenic factors have been identified, the molecules underlying perisynaptic astroctye-neuron contacts are unknown. Here we show that the $\gamma$-protocadherins ( $\gamma$-Pcdhs), a family of 22 neuronal adhesion molecules encoded by a single gene cluster, are also expressed by astrocytes and localize to their perisynaptic processes. Using cocultures in which either astrocytes or neurons are $P c d h$ - $\gamma$-null, we find that astrocyte-neuron $\gamma$-Pcdh contacts are critical for synaptogenesis in developing cultures. Synaptogenesis can eventually proceed among neurons cocultured with $P c d h-\gamma$-null astrocytes, but only if these neurons themselves express the $\gamma$-Pcdhs. Consistent with this, restricted mutation of the Pcdh- $\gamma$ cluster in astrocytes in vivo significantly delays both excitatory and inhibitory synapse formation. Together, these results identify the first known contact-dependent mechanism by which perisynaptic astrocyte processes promote synaptogenesis.

\section{Introduction}

Astrocytes, glial cells that greatly outnumber neurons in the mammalian brain, elaborate processes that are closely apposed to synaptic terminals. Astrocytes modulate synaptic transmission by buffering extracellular ions, controlling neurotransmitter levels, and releasing neuroactive molecules (Barres, 2008; Theodosis et al., 2008). Recent work has shown that astrocytes are also critical mediators of synaptogenesis (Pfrieger and Barres, 1997; Mauch et al., 2001; Ullian et al., 2001; Christopherson et al., 2005; Colón-Ramos et al., 2007; Barker et al., 2008).

Astrocytes promote synapse formation in part through their release of secreted molecules. Retinal ganglion cells (RGCs) cultured with astrocytes form many more synapses than those cultured alone, an effect that can be partially mimicked by culturing with astrocyte conditioned media (ACM) (Christopherson et al., 2005). The major synaptogenic component of ACM has been identified as the thrombospondins (TSPs), the addition of which stimulates the formation of synapses that are presynaptically active but postsynaptically silent (Christopherson et al., 2005). RGCs cultured in the absence of astrocytes are also deficient in cholesterol, which can be released by astrocytes, and addition of cholesterol to RGC cultures has been reported to result in an increase in synaptic puncta and in synaptic efficacy (Mauch et al., 2001).

Electron micrographs reveal extensive apposition of astrocyte processes and synaptic terminals in vivo (Ventura and Harris, 1999), and direct contact between neurons and astrocytes in vitro

Received June 15, 2009; revised July 17, 2009; accepted Aug. 1, 2009.

This work was supported by grants from the Roy J. Carver Charitable Trust and from the National Institutes of Health (R01 NS055272) to J.A.W. and by a predoctoral fellowship from the American Heart Association to A.M.G. We thank Leah Fuller and Mark Lobas for technical assistance, and Drs. Joshua Sanes and Michael Dailey for helpful discussions.

Correspondence should be addressed to Joshua A. Weiner, Department of Biology, Neuroscience Graduate Program, The University of lowa, 143 Biology Building, lowa City, IA 52242. E-mail: joshua-weiner@uiowa.edu. DOI:10.1523/JNEUROSCI.2818-09.2009

Copyright $\odot 2009$ Society for Neuroscience ～0270-6474/09/2911723-09\$15.00/0 is also critical for proper synaptogenesis. RGCs cultured from young (E17) rats make very few synapses when an astrocyte feeder layer is separated by a filter, but exhibit robust synaptogenesis when cultured directly atop astrocytes (Barker et al., 2008). Direct contact is required to make the RGCs competent to receive synaptic inputs: Young RGCs grown in the absence of astrocyte contacts can form presynaptic specializations on older RGCs, but are unable to develop postsynaptic specializations (Barker et al., 2008). Contact between astrocytes and single hippocampal neurons in microisland cultures also increases the number of autapses globally, through a mechanism involving PKC signaling (Hama et al., 2004). The identities of the molecules that mediate the specific perisynaptic astrocyte-neuron contacts observed in vivo remain unknown.

We showed previously that the $\gamma$-protocadherins ( $\gamma$-Pcdhs), a family of 22 neuronal adhesion molecules, are critical for nervous system development. Mice in which the $P c d h-\gamma$ gene cluster has been deleted $\left(P c d h-\gamma^{d e l / d e l}\right)$ die shortly after birth and exhibit massive interneuron apoptosis and reduced synapse density in the spinal cord (Wang et al., 2002b; Weiner, 2006; Prasad et al., 2008). Blocking apoptosis fails to rescue synapse density in $P c d h-\gamma$ mutant neonates, indicating a primary role for the $\gamma$-Pcdhs in synaptogenesis (Weiner et al., 2005). Despite this, only a portion of all $\gamma$-Pcdh protein is found at the synapse: much of it localizes perisynaptically or within tubulovesicular structures within nerve terminals (Wang et al., 2002b; Phillips et al., 2003). Here, we have tested the hypothesis that the observed perisynaptic $\gamma$-Pcdh might be localized within astrocyte processes, and that the synaptogenesis-promoting activity of the $\gamma$-Pcdhs might be mediated, in part, by astrocyte-neuron contacts.

\section{Materials and Methods}

Mouse strains. $P c d h-\gamma^{d e l}, P c d h-\gamma^{f u s g}$ (Wang et al., 2002b), and $P c d h-\gamma^{f c o n 3}$ alleles (Prasad et al., 2008) have been described previously. GFAP-Cre (Zhuo et al., 2001), Actin-Cre (Lewandoski et al., 1997), and Z/EG (Novak et 
al., 2000) mouse lines were obtained from The Jackson Laboratory (Bar Harbor, ME). The Cre-ER mouse line used in most studies (Guo et al., 2002) was obtained from Dr. Joshua Sanes; a second, similar Cre-ER line (Hayashi and McMahon, 2002) was obtained from The Jackson Laboratory.

Tamoxifen injections. Mice were injected intraperitoneally with 180 $\mathrm{mg} / \mathrm{kg}$ tamoxifen (Sigma Aldrich) diluted in corn oil to a final volume of $250 \mu$ l. Five daily injections were performed beginning when the animals were $14 \mathrm{~d}$ of age. When mice reached 1 month of age, spinal cord and hippocampal tissues were isolated and prepared for immunofluorescence as described below.

Astrocyte-neuron cocultures. Spinal astrocyte cultures were prepared from P0-P2 animals. Spinal cords were dissected from the column, chopped into small sections, and incubated in $0.05 \%$ trypsin/EDTA (Invitrogen) for $30 \mathrm{~min}$ at $37^{\circ} \mathrm{C}, 5 \% \mathrm{CO}_{2}$. Trypsin was quenched by adding $2 \mathrm{ml}$ of growth media (MEM, 15\% fetal calf serum, $0.6 \%$ glucose, $1 \times$ Primocin [Invivogen]) and the tissue was transferred to $1 \mathrm{ml}$ of fresh growth media. The tissue was then triturated 10-20 times through a fire-polished Pasteur pipette before plating in tissue culture dishes. Each individual spinal cord was prepared separately and plated in one well of a six-well culture dish and maintained in growth media. Once the astrocytes reached confluence, they were expanded to a $10 \mathrm{~cm}$ dish. Astrocytes were allowed to reach confluence again before being trypsinized and plated at the same relative density on glass coverslips that had been coated sequentially with poly-L-lysine (Sigma) and EHS laminin (Invitrogen); cultures were then maintained for $3 \mathrm{~d}$ in growth media. Twenty-four hours before adding spinal interneurons, astrocytes cultures were changed to Neurobasal medium containing B27 supplements (Invitrogen), glutaMAX (Invitrogen) and Primocin. Interneurons were prepared from E13 mice essentially as described previously (Weiner et al., 2005) and plated directly onto established astrocyte cultures at 400,000 cells/ coverslip. After $24 \mathrm{~h}$, and at subsequent $48 \mathrm{~h}$ intervals, the media was changed to Neurobasal/B27/glutaMAX medium without Primocin. For some cocultures, astrocyte monolayers were fixed in $2 \%$ paraformaldehyde (PFA) for $30 \mathrm{~min}$ at room temperature and extensively washed in PBS for $3 \mathrm{~h}$ before plating neurons as described above.

$A C M$. Confluent spinal astrocyte cultures were maintained in Neurobasal/B27/glutaMAX media for $3 \mathrm{~d}$, after which media was removed and concentrated to $10 \times$ using Vivaspin columns with a 3000 MW cutoff (Millipore). ACM was delivered at a final concentration of $2 \times$ diluted in Neurobasal/B27/glutaMAX media at each media change. Western blot analysis was performed on equal volumes of $1 \times$ ACM collected from wild type and $P c d h-\gamma^{d e l / d e l}$ cultures with an antibody directed against thrombospondin-2 (Becton-Dickinson; Franklin Lakes, NJ, 1:500) using standard methods.

Immunofluorescence. Tissue was prepared using two different methods: 1) Tissue was dissected, immersed in OCT compound (Sakura; Torrance, CA) and snap frozen in isopentane cooled in a dry ice/ethanol bath; or 2) tissue was fixed in $4 \%$ PFA either by transcardial perfusion followed by immersion overnight at $4{ }^{\circ} \mathrm{C}$ (adult animals) or direct immersion for $2 \mathrm{~h}$ at $4^{\circ} \mathrm{C}$ (embryonic and neonatal animals). Cryostat sections were cut at $6-12 \mu \mathrm{m}$ and fresh-frozen sections were fixed in either $100 \%$ methanol for $10 \mathrm{~min}$ at $-20^{\circ} \mathrm{C}$ or $2 \% \mathrm{PFA} / 0.2 \%$ Triton $\mathrm{X}-100$ for $30 \mathrm{~min}$ at room temperature. Cultures were fixed in $100 \%$ methanol for $10 \mathrm{~min}$ at $-20^{\circ} \mathrm{C}$ or in $2 \%$ PFA for $30 \mathrm{~min}$ at room temperature. Immunostaining was performed as described previously (Weiner et al., 2005). $\gamma$-Pcdh localization was examined by two methods: (1) using tissue from $P c d h$ $\gamma^{\text {fusg }}$ or $P c d h-\gamma^{f c o n 3}$ knock-in animals with antibodies directed against GFP to detect the third constant exon-GFP fusion; or (2) using anti- $\gamma$ Pcdh constant domain monoclonal or polyclonal antibodies. As we demonstrated previously (Wang et al., 2002b), both methods produced similar staining patterns that were completely specific: no staining using anti-GFP antibodies was observed in wild-type (WT) tissues, and no staining using anti-constant domain antibodies was observed in Pcdh$\gamma^{\text {del/del }}$ tissues (supplemental Fig. S1, available at www.jneurosci.org as supplemental material). A complete list of antibodies used is presented in supplemental Table 1 online.

In situ hybridization. In situ hybridizations were performed as described (Wang et al., 2002b), using antisense probes directed against the $P c d h-\gamma$ constant exons and against GFAP. Briefly, tissue was snap-frozen on dry ice-ethanol-cooled isopentane in OCT compound. Twenty mi- cron cryostat sections were cut, thawed onto charged slides, and postfixed for $5 \mathrm{~min}$ at room temperature. Sections were rinsed and then immersed in acetylation solution $\left(295 \mathrm{ml}\right.$ of $\mathrm{H}_{2} \mathrm{O}, 4 \mathrm{ml}$ of triethanolamine, $0.525 \mathrm{ml}$ of concentrated $\mathrm{HCl}, 0.75 \mathrm{ml}$ of acetic anhydride) for 10 $\mathrm{min}$ at room temperature. After this, slides were rinsed and overlain with hybridization solution ( $50 \%$ formamide, $5 \times$ SSC, $5 \times$ Denhardt's solution, $250 \mu \mathrm{g} / \mathrm{ml}$ yeast tRNA, $500 \mu \mathrm{g} / \mathrm{ml}$ salmon sperm DNA, $50 \mu \mathrm{g} / \mathrm{ml}$ heparin) at room temperature for $1 \mathrm{~h}$ before being hybridized overnight at $65^{\circ} \mathrm{C}$ in hybridization solution containing $\sim 400 \mathrm{ng} / \mathrm{ml}$ fluoresceinUTP and/or digoxigenin-UTP-labeled riboprobe. The next day, slides were washed for several hours at $65^{\circ} \mathrm{C}$ in $0.2 \times \mathrm{SSC}$, rinsed at room temperature in TBS, and blocked for $1 \mathrm{~h}$ with $0.2 \%$ Blocking Reagent (Roche) in TBS. Probes were detected by overnight incubation at $4^{\circ} \mathrm{C}$ with anti-digoxigenin or anti-fluorescein antibodies (1:1000) conjugated to peroxidase (Roche), followed by amplification using the TSA Plus system (Perkin-Elmer Life Sciences) according to manufacturer's protocols. Fluorescein-labled probes were amplified using FITC-tyramide, and digoxigenin-labeled probes were amplified using Cy3 tyramide. Double-labeled hybridizations were detected sequentially; peroxidase from the first antibody was inactivated with $0.3 \% \mathrm{H}_{2} \mathrm{O}_{2}$ in TBS for $40 \mathrm{~min}$ before beginning the second antibody incubation. No signal was observed when hybridization was performed on $P c d h-\gamma^{d e l / d e l}$ tissues.

Reverse-transcriptase PCR. Reverse-transcriptase (RT)-PCR was performed on RNA isolated from spinal astrocyte cultures with RNAqueous (Ambion). First-strand cDNA synthesis using M-MLV reverse transcriptase (Invitrogen) used standard conditions. To detect the $22 \gamma$-Pcdhs, we used a previously published primer set (Prasad et al., 2008) consisting of a common reverse primer in the $3^{\prime}$ UTR of $\mathrm{C}$ exon 3 and 22 distinct forward primers specific to each of the $\mathrm{V}$ exons. Primer sequences are available on request. Cycling parameters were: $94^{\circ} \mathrm{C}, 1 \mathrm{~min} ; 55^{\circ} \mathrm{C}, 1 \mathrm{~min}$; $72^{\circ} \mathrm{C}, 3 \mathrm{~min}$ for 30 cycles.

Image collection and analysis. Images were collected directly into Photoshop (Adobe;) using $10 \times, 20 \times$, or $63 \times$ PlanApo objectives on a Leica DM5000B epifluorescense microscope or into Leica LCS software using a $63 \times$ PlanApo objective on a Leica SP2 AOBS laser-scanning confocal microscope. To investigate the perisynaptic localization of $\gamma$-Pcdh in Cre-ER; Pcdh- $\gamma^{f c o n 3 /+}$ mice, confocal $z$-stacks were collected with a $4 \times$ digital zoom factor and cross-sections reconstituted in LCS software. The images were adjusted for brightness and contrast and filtered with a 0.5 pixel Gaussian blur. Synapses were quantified from immunostained cryostat sections using single confocal planes collected with a $2 \times$ digital zoom factor in the intermediate gray ventral and lateral to the central canal. Circular fields, $50 \mu \mathrm{m}$ in diameter and centered on a GFAPpositive astrocyte, were extracted from the raw images and adjusted identically for contrast and brightness. Using the Photoshop "multiply" calculation, synapses, defined as apposed and partially overlapping red and green puncta, were extracted, inverted to black, and hand-counted. In vitro synapses were quantified using the Puncta Analyzer plug-in in NIH Image/J as described (Christopherson et al., 2005). MAP2 images collected from representative microscope fields were thresholded and the area of MAP2 immunolabeling was quantified using the measurement tools in NIH Image/J. All quantitative data were analyzed in Prism (GraphPad Software) using two-way ANOVAs with post hoc Bonferroni tests to compare genotypes or culture conditions at each time point. Student's $t$ tests were used in instances in which only 2 means were compared.

\section{Results}

\section{Astrocytes express multiple $\gamma$-Pcdhs}

We focused on the developing spinal cord, which matures earlier than the brain and in which the consequences of $\gamma$-Pcdh disruption have best been characterized (Wang et al., 2002b; Weiner et al., 2005; Prasad et al., 2008). The 6 extracellular cadherin repeats, transmembrane domain, and proximal cytoplasmic domain of each $\gamma$-Pcdh protein is encoded by one of 22 large "variable" (V) exons, each of which is spliced to three "constant" (C) exons that encode a shared 125-amino acid C-terminal domain (Wu and Maniatis, 1999; Tasic et al., 2002; Wang et al., 2002a). Using an in situ hybridization (ISH) probe against the $\mathrm{C}$ exons, we found 
A
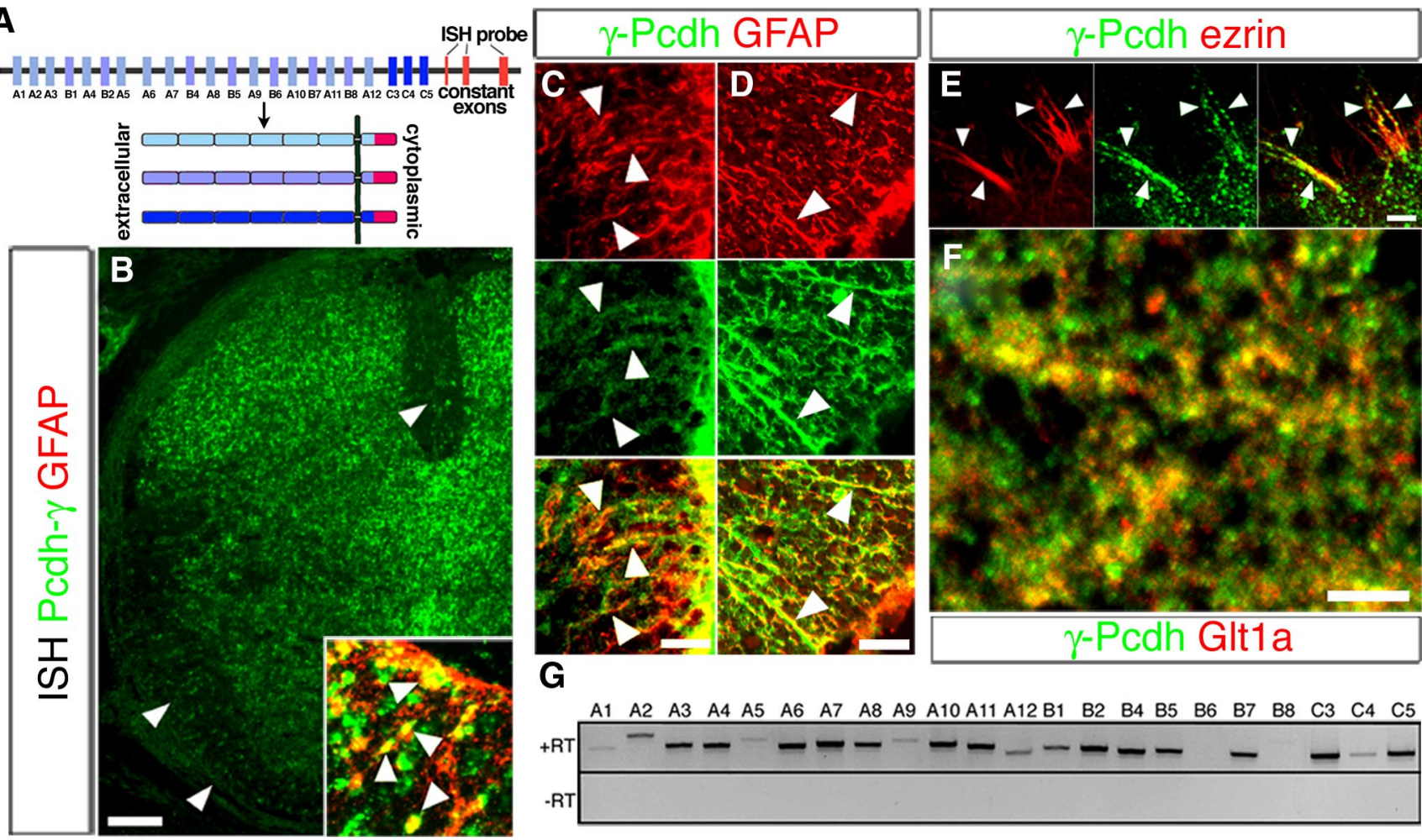

G

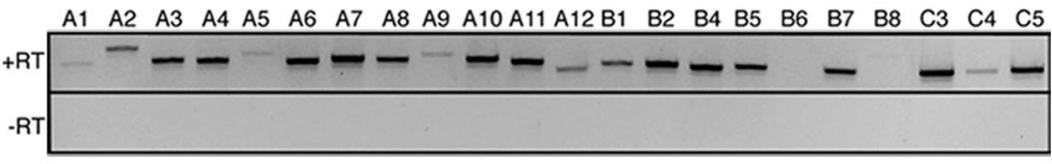

Figure 1. Astrocytes express multiple $\gamma$-Pcdhs. In situ hybridization (ISH) using a $P$ cdh- $\gamma$ C exon riboprobe (diagrammed in $\boldsymbol{A}$ ) revealed expression in cells of the white matter (arrowheads) as well as the gray matter in neonatal spinal cord ( $\boldsymbol{B})$. Double-labeling with a GFAP riboprobe indicated that many of these cells were astrocytes ( $\boldsymbol{B}$, inset shows higher-magnification view). Immunofluorescence confirmed $\gamma$-Pcdh protein expression by GFAP-positive astrocytes in neonatal $(\boldsymbol{C})$ and adult $(\boldsymbol{D})$ spinal cords. Immunostaining of cultured astrocytes $(\boldsymbol{E})$ and spinal cord sections $(\boldsymbol{F})$ demonstrated that $\gamma$-Pcdh proteins colocalize with ezrin and Glt-1a, both markers of perisynaptic astrocyte processes. Reverse-transcriptase (RT)-PCR analyses detected expression of 20 of the 22 possible $P$ Cdh- $\gamma$ spliced transcripts in cultured spinal cord astrocytes (G). Scale bars: $\boldsymbol{B}, 100 \mu \mathrm{m}$ (inset, $10 \mu \mathrm{m}$ ); $\boldsymbol{C}, \boldsymbol{D}, 25 \mu \mathrm{m} ; \boldsymbol{E}, 3 \mu \mathrm{m} ; \boldsymbol{F}, 4 \mu \mathrm{m}$.

that, in addition to ubiquitous expression in the gray matter, $P c d h-\gamma$ transcripts are expressed by cells in the white matter of the neonatal spinal cord (Fig. $1 B$ ). Double-label fluorescent ISH (Fig. $1 B$, inset) and immunofluorescence in both neonatal and adult spinal cord (Fig. 1C,D) showed that many of these cells were GFAP-positive astrocytes, which extend radial processes that contain $\gamma$-Pcdhs. Coexpression of $P c d h-\gamma$ genes with the PLP gene, a marker for oligodendrocytes, was not observed in doublelabel fluorescent ISH to neonatal sections (data not shown). GFAP-positive astrocytes in the gray matter also appeared to contain $\gamma$-Pcdh proteins, but the ubiquitous localization of neuronal $\gamma$-Pcdhs throughout the neuropil made it difficult to ascertain their subcellular localization (see below, and Fig. 2, for subsequent analyses addressing this). Astrocytes cultured from the neonatal spinal cord (Fig. $1 G$ ) and cortex (data not shown) expressed multiple $P c d h-\gamma$ genes, with 20 of the 22 expected spliced transcripts detectable by RT-PCR of astrocytic RNA.

\section{$\gamma$-Pcdhs localize to perisynaptic astrocyte processes}

In astrocyte cultures, $\gamma$-Pcdh proteins were concentrated at cellcell junctions and in small, filopodia-like processes that also contained ezrin (Fig. 1E), a marker of perisynaptic astrocyte processes in vivo (Derouiche and Frotscher, 2001). $\gamma$-Pcdh proteins colocalized extensively with another marker of these processes, the glutamate transporter Glt-1a (Rothstein et al., 1994; Cholet et al., 2002), in the spinal cord in vivo (Fig. $1 F$ ). To more clearly determine the subcellular localization of astrocytic $\gamma$-Pcdhs, we developed a genetic method using a conditional mutant allele, termed $P c d h-\gamma^{f c o n} 3$, in which a C exon 3-GFP fusion is flanked by loxP sites (Fig. $2 A$ ). Ubiquitous homozygous excision of the $P c d h-\gamma^{f c o n 3}$ allele results in a phenocopy of $P c d h-\gamma^{d e l / d e l}$ null mutants (Prasad et al., 2008); because of the GFP fusion, heterozygous $P c d h-\gamma^{f c o n 3 /+}$ mice can also be used to report on Cre activity in phenotypically normal tissues.

We crossed $P c d h-\gamma^{f c o n} 3$ mice with a transgenic line expressing a Cre-estrogen receptor (Cre-ER) fusion protein that can localize to the nucleus only on binding of the synthetic ligand tamoxifen (Guo et al., 2002). After tamoxifen injection of Cre-ER; Pcdh- $\gamma^{f c o n 3 /+}$ heterozygous mice, we found that the GFP-tagged exon was removed from almost all cells in the spinal cord. A small number of isolated cells in the gray matter, however, escaped excision, and thus retained GFP-tagged $\gamma$-Pcdh proteins (Fig. 2B). These cells had the morphology of protoplasmic astrocytes, and expressed GFAP (Fig. 2C) but not the neuronal marker NeuN (see supplemental Fig. S2, available at www.jneurosci.org as supplemental material). By using confocal microscopy to examine sections from these heterozygous mice stained with antibodies against GFP and excitatory and inhibitory synaptic markers, we thus could visualize astrocytic $\gamma$-Pcdhs in isolation and unambiguously determine their relationship to synapses in phenotypically normal tissue.

These analyses confirmed that astrocytic $\gamma$-Pcdhs were often concentrated in puncta directly adjacent to synaptic contacts in the spinal cord (Fig. $2 F, G$ ), as well as in the hippocampus, where a subset of astrocytes also escaped Cre excision (supplemental Fig. S2, available at www.jneurosci.org as supplemental material). These perisynaptic puncta were closely apposed to both excitatory (86\% of 198 apposed synaptic puncta) and inhibitory ( $87 \%$ of 247 apposed synaptic puncta) synapses and often appeared to wrap around or interdigitate between them (Fig. $2 \mathrm{~F}-\mathrm{H}$ ). Similar analyses in which Cre-ER; Pcdh- $\gamma^{f c o n 3 /+}$ sections were triple-immunostained 


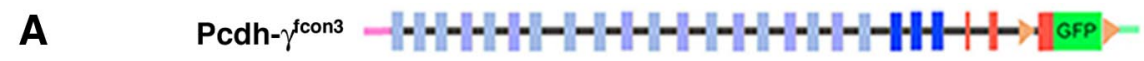
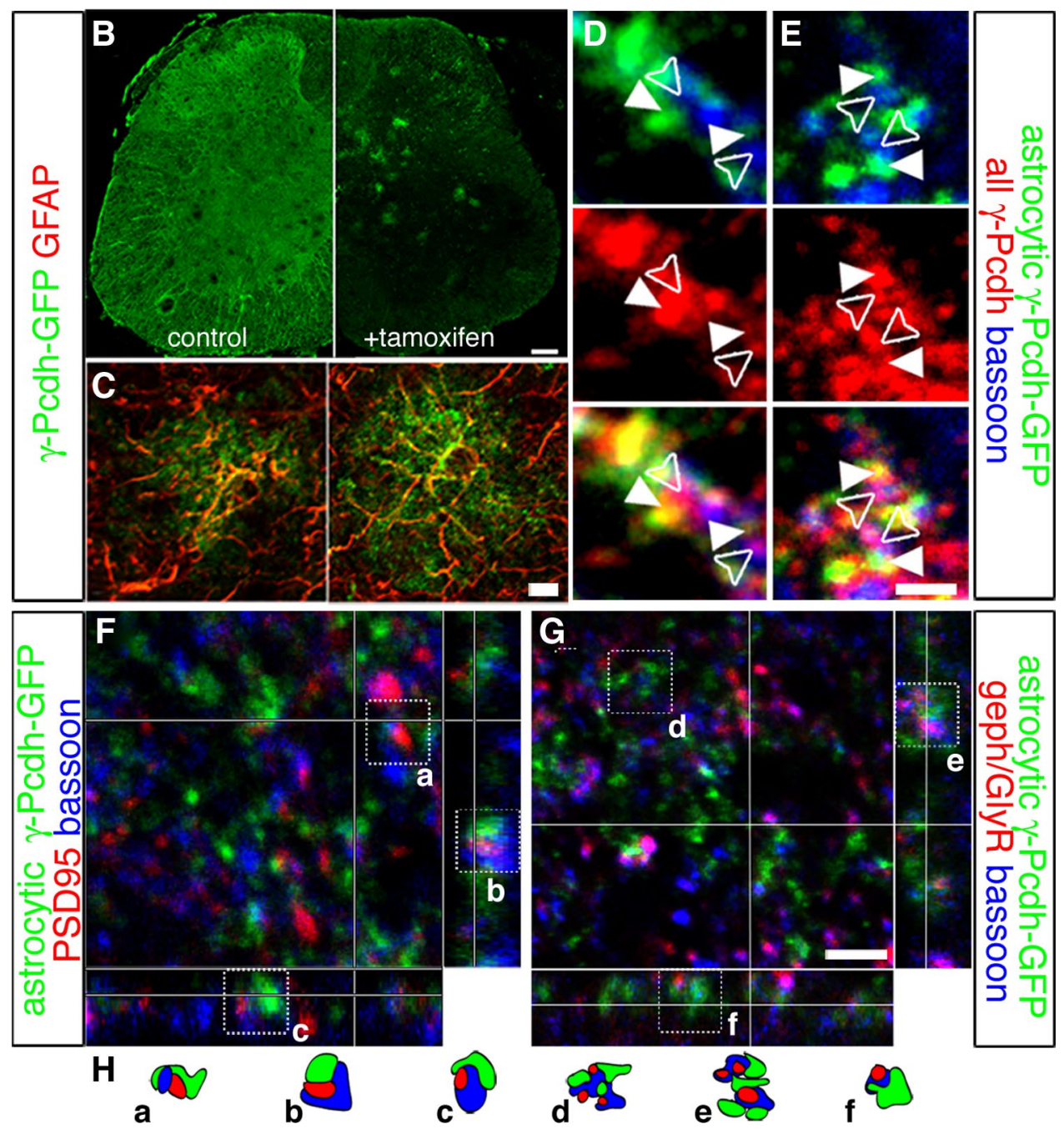

Figure 2. $\quad \gamma$-Pcdh proteins localize to perisynaptic astrocyte processes. $A$, Schematic diagram of the $P$ cdh- $\gamma^{\text {fcon3 } 3}$ conditional mutant allele, in which a GFP-fused C exon 3 is flanked by loxP sites. The A-C subfamilies of $V$ exons are in shades of blue, and the 3 C exons are in red. $B, \operatorname{In} C r e-E R ; P c d h-\gamma^{\text {fconz/+ }}$ mice injected with tamoxifen (B, right), Cre excision resulted in loss of the ubiquitous $\gamma$-Pcdh-GFP immunofluorescence observed in uninjected or Cre-ER-negative mice ( $\boldsymbol{B}$, left). GFP-tagged $\gamma$-Pcdhs remained detectable, however, in a small number of GFAP-positive gray matter protoplasmic astrocytes $(\boldsymbol{B})$. C, A higher-magnification image of a separate double-immunostained cryosection is shown. $\boldsymbol{D}, \boldsymbol{E}$, Confocal analysis of immunostained $\left(r e-E R\right.$; $P$ cdh- $\gamma^{\text {fcon } 3 /+}$ spinal cord sections demonstrated that astrocytic $\gamma$-Pcdh puncta (stained for both GFP and $\gamma$-Pcdh; closed arrowheads; yellow in merged images) can localize directly adjacent to neuronal $\gamma$-Pcdh at synaptic puncta (stained for both bassoon and $\gamma$-Pcdh; open arrowheads, magenta in merged images). $F, G$, Immunostaining for GFP and presynaptic and postsynaptic markers confirmed that astrocytic $\gamma$-Pcdhs were frequently apposed to and/or wrapped around synaptic terminals. Large images are of single planes from $z$-stacks; perpendicular cross-sections through the entire $z$-stacks at locations indicated by white lines are shown at right and below. $\boldsymbol{H}$, Tracings of several such perisynaptic contacts for the indicated boxes in $\boldsymbol{F}$ and $\boldsymbol{G}$ are presented. Scale bars: $\boldsymbol{B}, 100 \mu \mathrm{m} ; \boldsymbol{C}, 6 \mu \mathrm{m} ; \boldsymbol{D}, \boldsymbol{E}, 1 \mu \mathrm{m} ; \boldsymbol{F}, \boldsymbol{G}, 2 \mu \mathrm{m}$.

with antibodies against GFP (to detect astrocytic $\gamma$-Pcdhs), the constant domain (to detect all $\gamma$-Pcdhs) (see supplemental Fig. S1, available at www.jneurosci.org as supplemental material), and the presynaptic marker bassoon identified $\gamma$-Pcdh-positive synaptic puncta adjacent to astrocytic $\mathrm{GFP} / \gamma$-Pcdh-double-positive puncta (Fig. 2D,E). Together, these results indicate that the $\gamma$-Pcdhs are appropriately situated to mediate adhesive contacts between neuronal synapses and perisynaptic astrocyte processes.

\section{Astrocytic $\boldsymbol{\gamma}$-Pcdhs are critical for synaptogenesis in} developing neurons in vitro

To determine whether astrocytic $\gamma$-Pcdhs play a role in synaptogenesis, we began by modifying a coculture system used in our previous work, in which embryonic spinal interneurons grow atop a lawn of astrocytes, differentiating and forming fully mature synapses over a period of $\sim 9 \mathrm{~d}$ (Weiner et al., 2005). Instead of mixed cultures prepared from the same animals, we separately established a monolayer of neonatal spinal cord astrocytes, on which interneurons from E13 spinal cords were subsequently plated: this allowed us to independently control the genotype of each cell type. Neurons do not survive the astrocyte preparation (data not shown), obviating any concern that interneurons of the undesired genotype might be carried over in cocultures. The converse complication-carrying over astrocytes when plating the interneuron preparation-is, however, possible. Therefore, to ensure that no astrocytes of the wrong genotype were present, we established test cocultures of WT astrocytes with neurons from $P c d h-\gamma^{\text {fusg/+ }}$ mice, which harbor the same C exon 3-GFP fusion as the Pcdh- $\gamma^{f c o n 3}$ allele (Wang et al., 2002b) (Fig. 3A). In these cultures, only TuJ1-positive neurons were GFP-positive, confirming that our method allowed for complete separability of astrocyte and neuron genotype. We also confirmed that $\gamma$-Pcdhs 


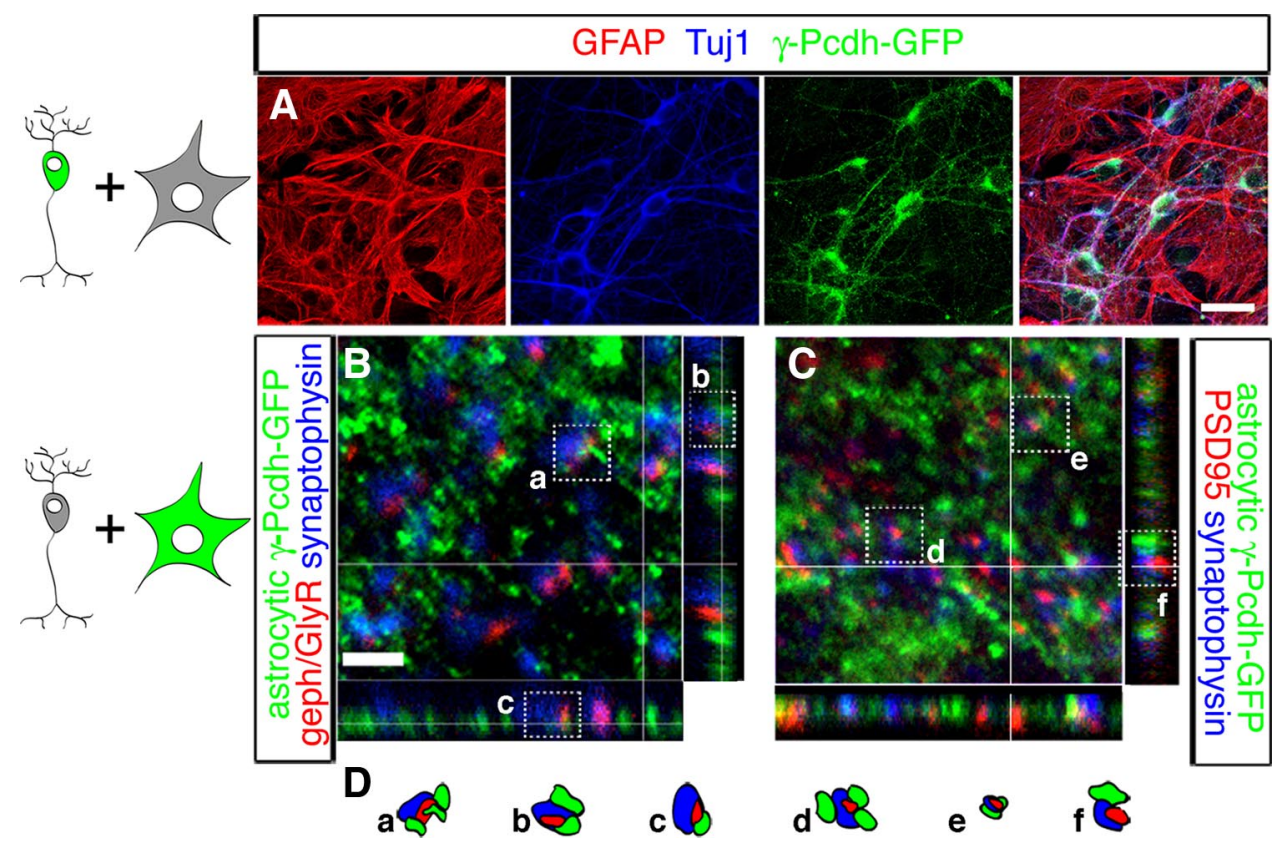

Figure 3. A-C, Cocultures of spinal cord interneurons and astrocytes of distinct genotypes. Cocultures of neurons from Pcdh- $\gamma^{\text {fusg }}$ mice with wild type astrocytes were immunostained with antibodies against the indicated proteins $(\boldsymbol{A})$. Only TuJ1-positive neurons expressed GFP-tagged $\gamma$-Pcdhs, indicating that the coculture system allowed for effective separability of neuron and astrocyte genotypes with no carryover. Cocultures of wild type neurons with astrocytes from $P c d h-\gamma^{\text {fusg }}$ mice stained for GFP and inhibitory $(\boldsymbol{B})$ or excitatory $(\boldsymbol{C})$ synaptic markers demonstrated that astrocytic $\gamma$-Pcdhs localized to many sites adjacent to synaptic terminals in vitro, as observed in vivo. Large images are of single planesfrom z-stacks; perpendicular cross-sections through the entirez-stacks at locations indicated by white lines are shown at right and below. $\boldsymbol{D}$, Tracings of several such perisynaptic contacts for the indicated boxes in $\boldsymbol{B}$ and $\boldsymbol{C}$ are presented. Scale bars: $\boldsymbol{A}, 20 \mu \mathrm{m} ; \boldsymbol{B}, \boldsymbol{C}, 2 \mu \mathrm{m}$.

can localize to perisynaptic processes in these cultures as they do in vivo, by conversely coculturing wild-type neurons with astrocytes prepared from $P c d h-\gamma^{f u s g /+}$ mice. Confocal microscopy of these cocultures demonstrated the presence of astrocytic $\gamma$-Pcdh puncta adjacent to many excitatory and inhibitory synapses (Fig. $3 B-D)$.

We then proceeded to establish cultures in which either astrocytes or neurons were prepared from null mutant $P c d h-\gamma^{d e l / d e l}$ mice (Wang et al., 2002b; Weiner et al., 2005; Prasad et al., 2008) ["astronull" (astrocyte-null) and "neuron-null," respectively], and compared the progression of synaptogenesis in these cultures to that in cocultures in which both cell types were normal ("control"). We observed no apparent differences in survival, growth rate, morphology, or GFAP expression between control and mutant astrocyte monolayers. Neuronal differentiation and neurite outgrowth, assessed here by immunostaining for the markers MAP2 (Fig. 4O) and $\mathrm{TuJ1}$ (data not shown) were also indistinguishable across all types of cultures, consistent with our extensive previous demonstration of normal neurogenesis, differentiation, and axon outgrowth in Pcdh$\gamma$-null mutant mice (Wang et al., 2002b; Prasad et al., 2008).

In control cultures, synapses (defined by apposition and partial overlap of presynaptic and postsynaptic puncta) were already numerous by $6 \mathrm{~d}$ in vitro (DIV) and increased in number by 9 DIV (Fig. $4 A, D, G, J, M, N$ ). In astro-null cultures, however, few synapses had formed by 6 DIV: excitatory synapses were reduced by $77 \%(n=22$ fields from 6 cultures; $p<0.001)$ and inhibitory synapses by $39 \%$ ( $n=33$ fields from 6 cultures; $p<0.001)$ (Fig. $4 B, H, M, N)$. By 9 DIV, synapse density in astro-null cultures recovered toward control levels, although inhibitory synapses were still significantly reduced in number (Fig. $4 E, K, M, N$ ). In neuron-null cultures, formation of both excitatory and inhibitory synapses was drastically reduced at both 6 DIV (by 67\% combined; total of $n=44$ fields from 12 cultures; $p<0.001$ ) and 9 DIV (by $80 \%$ combined; total of $n=44$ fields from 12 cultures; $p<0.001$ ) (Fig. $4 C, F, I, L-N)$. These results indicate that astro- cytic $\gamma$-Pcdhs are critical for synapse formation or stabilization in developing cultures, but that synaptogenesis can eventually proceed, provided that neurons themselves express the $\gamma$-Pcdhs.

\section{Astrocytic $\gamma$-Pcdh control of synaptogenesis is contact dependent}

Although the $\gamma$-Pcdhs can mediate homophilic adhesion in nonneural cell lines (Obata et al., 1995; Frank et al., 2005) it remains unclear whether this is their only mechanism of action in the nervous system. To ask whether loss of the $\gamma$-Pcdhs in astrocytes might have a secondary effect on their release of synaptogenic secreted factors, we collected tissue culture media that had been conditioned by either control (WT) or Pcdh- $\gamma$-null [knock-out (KO)] astrocytes. Astrocyte-conditioned media (ACM) from KO cultures contained levels of the known synaptogenic molecule TSP-2 (Christopherson et al., 2005) indistinguishable from that of WT ACM (Fig. 5A). Consistent with this, addition of concentrated WT or KO ACM produced a quantitatively similar promotion of synaptogenesis in control cultures (Fig. $5 B$ ), indicating that $P c d h-\gamma$ mutation does not disrupt astrocyte secretion of synaptogenic factors. Additionally, concentrated WT ACM was unable to completely rescue the reduced synapse density observed in astro-null cocultures (Fig. $5 C$ ), suggesting that $P c d h-\gamma$ mutation disrupts other, presumably contact-dependent, mechanisms.

To directly assess whether astrocytic $\gamma$-Pcdhs promote synaptogenesis in a contact-dependent manner, we prepared cocultures in which the astrocytic monolayer had been fixed with paraformaldehyde before addition of interneurons. We reasoned, as others have (Yamagata et al., 1995; Barker et al., 2008), that this manipulation should remove any differences in cell signaling or in release of secreted factors between WT and $\mathrm{KO}$ astrocytes. At 6 DIV in these cocultures, both excitatory and inhibitory synapses remained significantly reduced when astrocytes were $P c d h-\gamma$-null (Fig. 5D), further supporting a role for 

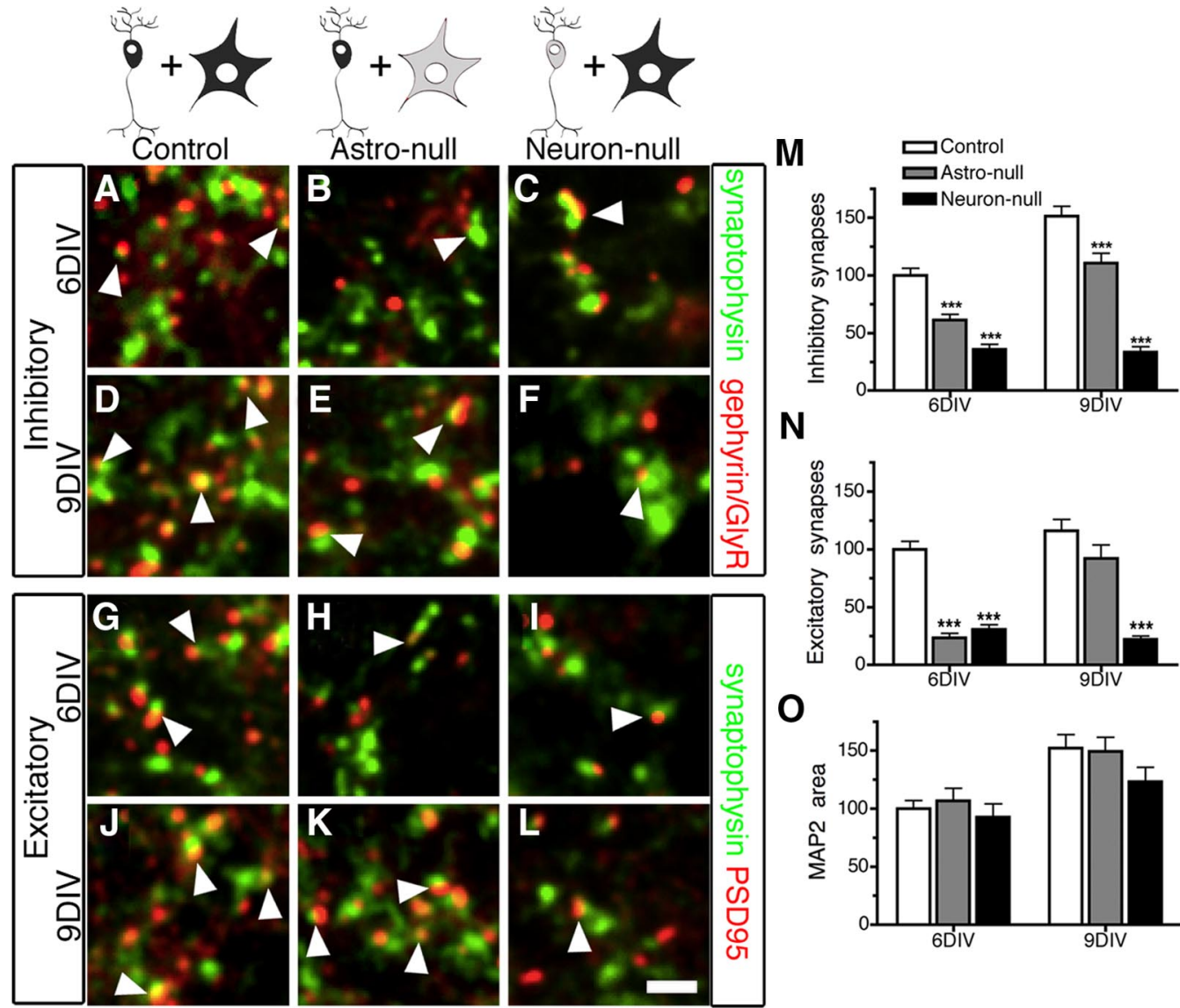

$\mathbf{N}$

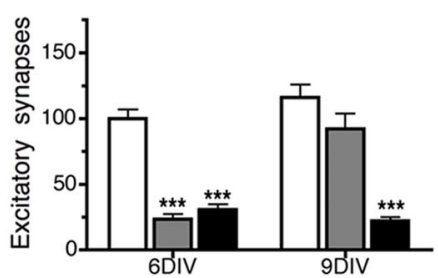

O

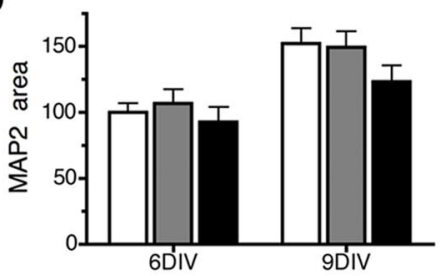

Figure 4. Astrocytic $\gamma$-Pcdhs are critical for synaptogenesis in developing neuronal cultures. Cocultures of embryonic spinal interneurons growing directly atop a confluent astrocyte monolayer were prepared such that either astrocytes (astro-null), neurons (neuron-null), or neither (control) were Pcdh- $\gamma$-null, and synaptogenesis monitored by quantifying the density of appositions of presynaptic and postsynaptic markers (i.e., the number of such synaptic contacts per a defined area) as neurons matured between 6 and $9 \mathrm{~d}$ in vitro (DIV). When astrocytes were mutant, the numbers of both excitatory and inhibitory synapses were significantly reduced at $6 \mathrm{DIV}(B, H, M, N)$ compared with control cultures $(A, G, M, N)$, although some recovery occurred by 9 DIV $(E, K, M, N)$. When neurons were mutant, synapse density was drastically reduced at both 6 and $9 \mathrm{DIV}(\boldsymbol{C}, F, I, L-N)$. Neuronal differentiation and survival, monitored by quantifying the area of MAP2 immunostaining, did not differ across the three culture conditions ( $\mathbf{0}$ ). Data in $\mathbf{M}-0$ are graphed as percentage of control values. Graphs in $\boldsymbol{M}-\mathbf{0}$ represent means \pm SEM from 22 to 44 fields from 6 cultures per time point. ${ }^{*} p<0.05 ;{ }^{* * *} p<0.001$. Scale bar (in $\boldsymbol{L}$ ): $\boldsymbol{A}-\boldsymbol{L}, 2 \mu \mathrm{m}$.
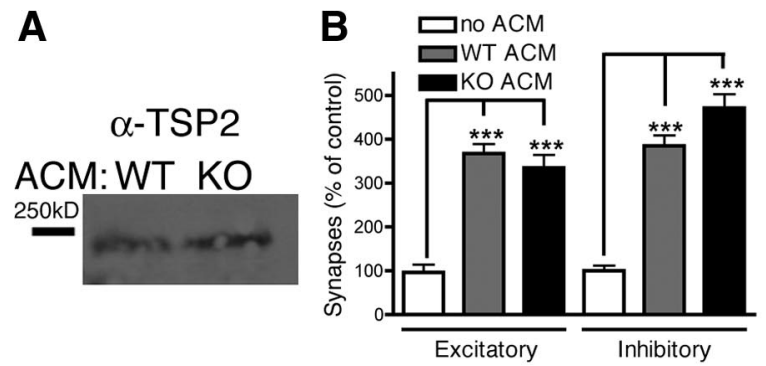

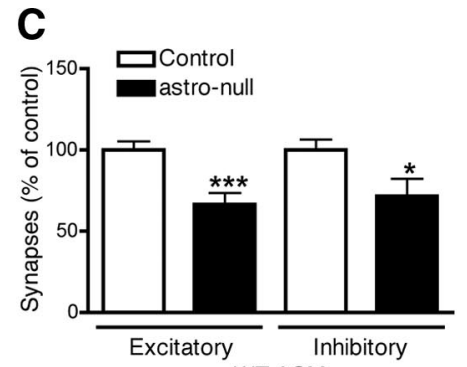

+ WT ACM

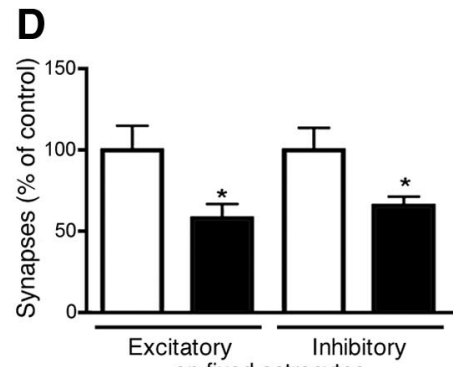

on fixed astrocytes

Figure 5. Astrocytic $\gamma$-Pcdhs regulate synaptogenesis through a contact-dependent mechanism. Astrocyte-conditioned medium (ACM) was collected from astrocyte cultures derived from WT or $P c d h-\gamma^{\text {del/del }}$ mice (KO). Western blot analysis of equal amounts of ACM showed no detectible difference between genotypes in the levels of thrombospondin-2 (TSP-2) secreted (A). Addition of concentrated WT or KO ACM to control cocultures over 6 DIV resulted in equally significant increases in the density of synapses (i.e., the number of synaptic contacts per a defined area) compared with control media ( $\boldsymbol{B})$; thus, WT and KO ACM are equivalent in their synaptogenic potency. Addition of concentrated WT ACM was unable to completely rescue the decrease in synapse density observed in astro-null cultures ( $\boldsymbol{C}$. Furthermore, the difference in synapse density between control and astro-null cocultures was maintained when the neurons were plated onto astrocytes that had been fixed with paraformaldehyde (D). Data are graphed as percentage of control values. Graphs represent means \pm SEM from 12 fields from 3 cultures per time point.

astrocytic $\gamma$-Pcdhs in providing contact-dependent cues for neurons that promote synapse formation or stabilization.

Astrocyte-restricted loss of the $\gamma$-Pcdhs results in delayed synaptogenesis in vivo

We next asked whether astrocytic $\gamma$-Pcdhs are important for synaptogenesis in vivo, by using the conditional $P c d h-\gamma^{f c o n 3}$ floxed allele and a transgenic line expressing the Cre recombinase under the control of the human GFAP promoter (Zhuo et al., 2001). Because many neurons are derived from radial glial precursors that may transiently express GFAP, this Cre line results in the excision of floxed alleles in some neurons as well as in astrocytes, and thus cannot be used for astrocyte-specific mutation in much of the CNS (data not shown; see Zhuo et al., 2001). There is evidence that neurons of the spinal cord, 


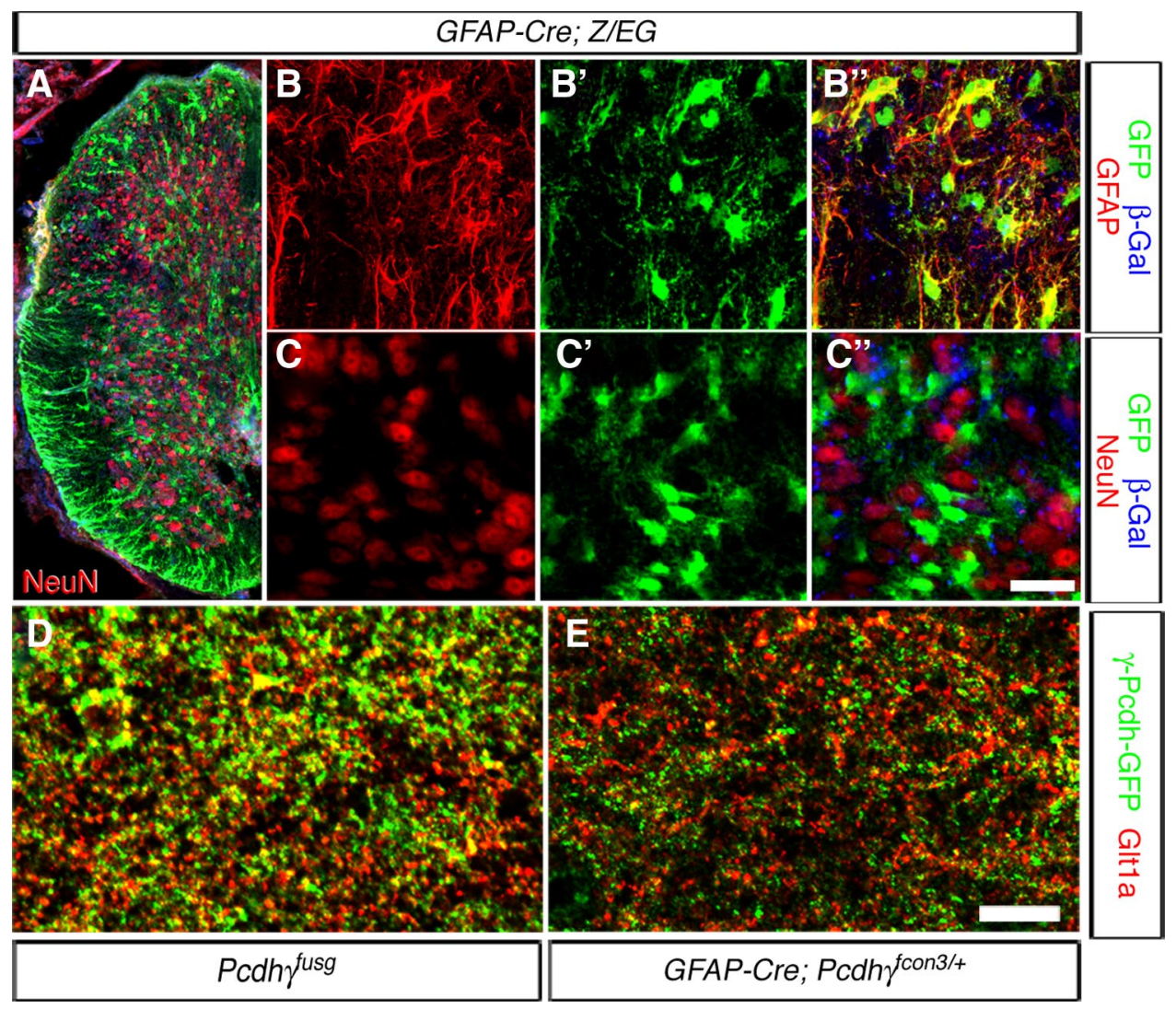

Figure 6. Astrocyte-restricted Pcdh- $\gamma$ mutation in vivo. In GFAP-Cre; Z/EG double transgenics ubiquitous expression of a $\beta$-galactosidase/neo fusion protein is replaced by that of GFP following Cre excision. GFAP-positive, but not NeuN-positive, cells express GFP, whereas NeuN-positive, but not GFAP-positive, cells express $\beta$-gal (A, higher-magnification images of gray matter in $B, C$, indicating that Cre is not expressed in neurons or their progenitors in the spinal cord. In the gray matter of GFAP-Cre; $P c d h-\gamma^{\text {fcon } 3 /+}$ spinal cords, there is reduced colocalization of $\gamma$-Pcdh-GFP with Glt1a, confirming that Cre recombinase is able to efficiently excise the floxed allele in astrocytes in these mice $(\boldsymbol{D}, \boldsymbol{E})$. Scale bars: $\boldsymbol{A}, 100 \mu \mathrm{m} ; \boldsymbol{B}, \boldsymbol{C}, 25$ $\mu \mathrm{m} ; \boldsymbol{D}, \boldsymbol{E}, 10 \mu \mathrm{m}$.

however, are not derived from GFAP + radial glial precursors (Barry and McDermott, 2005), which indicated that astrocyte-restricted excision might be attainable there.

We ascertained this by crossing GFAP-Cre mice with $Z / E G$ reporter mice, in which ubiquitous expression of a $\beta$-galactosidase/neo fusion protein is replaced by that of GFP following Cre excision (Novak et al., 2000) (Fig. 6A-C). In neonatal GFAPCre; $Z / E G$ double-transgenic spinal cords, immunostaining demonstrated that all GFP-positive cells were GFAP-positive and NeuN-negative, whereas $\beta$-galactosidase inclusions were detected only in NeuN-positive neurons (Fig. 6B,C). To confirm that the floxed allele was being efficiently excised in the astrocytes of GFAP-Cre; Pcdh- $\gamma^{\text {fcon } 3}$ mice, we immunostained double-heterozygous mice for the GFP-tagged constant exon and observed that nearly all colocalization of $\gamma$-Pcdh-GFP with the perisynaptic astrocyte marker Glt1a was lost (Fig. 6D,E). Having ascertained that GFAP-Cre effects astrocyte-specific excision in the developing spinal cord, we quantified synapses in sections

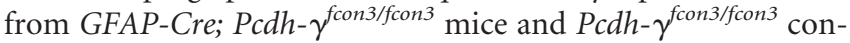
trol littermates at E15, E17, and P0, during the first major wave of synaptogenesis (May and Biscoe, 1975; Vaughn, 1989), the onset of which coincides with the appearance of GFAP+ astrocytes (Lee et al., 2003; Stolt et al., 2003; Barry and McDermott, 2005) (data not shown).

Because individual astrocytes are known to occupy nonoverlapping domains within which they contact multiple neuronal processes and synaptic terminals (Bushong et al., 2002; Halassa et al., 2007), we quantified synapses in circular fields $50 \mu \mathrm{m}$ in diameter and centered on GFAP-positive, $P c d h-\gamma$ mutant astrocytes (Fig. 7A-D). Both excitatory and inhibitory synapses were significantly reduced in fields from GFAP-Cre; Pcdh- $\gamma^{\text {fcon3/fcon } 3}$ mice compared with those from controls at E15 and E17 (Fig. $7 E)$. Importantly, no difference in the number of cleaved caspase3-positive cells was observed between astroctye-restricted mutants and controls, indicating that the reduction in synapse density was not caused by increased levels of neuronal apoptosis (supplemental Fig. S3, available at www.jneurosci.org as supplemental material). Similar to the recovery of synapse density observed in mature astro-null cocultures (Fig. $4 M, N$ ), we found that at $\mathrm{P} 0$, the number of synapses in fields from GFAP-Cre; $P c d h-\gamma^{f c o n 3 / f c o n 3}$ mice had reached control levels (Fig. 7E; supplemental Fig. $S 4 B, D$, available at www.jneurosci.org as supplemental material). This astrocyte-restricted mutant result contrasts with our previous experiments using $P c d h-\gamma^{d e l / d e l} ; B a x^{-/-}$mice (Weiner et al., 2005; Prasad et al., 2008), in which synapse density remains low at $\mathrm{P} 0$. Consistent with this, and with our coculture experiments (Fig. 4), we also found a significant reduction in synapse density persisted at P0 in Actin-Cre; $P c d h-\gamma^{f c o n 3 / f c o n} 3$ mice, in which both neurons and astrocytes are mutant (Fig. 7E; supplemental Fig. $\mathrm{S} 4 C, D$, available at www.jneurosci.org as supplemental material). The remarkable congruence between these in vivo data and our coculture experiments provides strong support for developmentally regulated roles of both astrocytic and neuronal $\gamma$-Pcdhs in the control of synaptogenesis. 

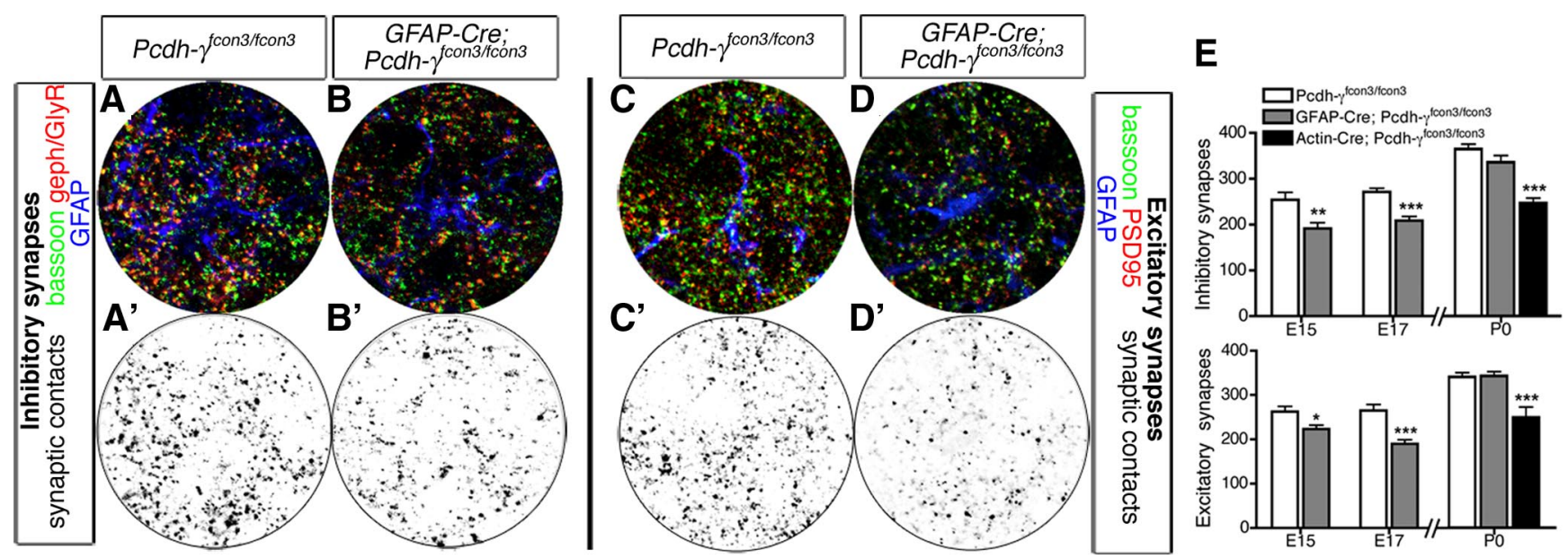

Figure 7. Astrocytic $\gamma$-Pcdhs control synaptogenesis in the embryonic spinal cord in vivo. Quantification of immunostained synapses was performed on $50 \mu m$ circular fields imaged in spinal cord sections from GFAP-Cre; Pcdh- $\gamma^{\text {fcon3/fcon3 }}$ astrocyte-restricted mutants and controls at E15, E17 (A-D), and P0 (see supplemental Fig. S4, available at www.jneurosci.org as supplemental material). In $\boldsymbol{A}^{\prime}-\boldsymbol{D}^{\prime}$, the yellow overlap between the red and green channels has been extracted and converted in Adobe Photoshop to black for clarity. $\boldsymbol{E}$, Synapse density (i.e., the number of such synaptic contacts per $50 \mu \mathrm{m}$ circle) was significantly reduced in GFAP-Cre; $P$ cdh- $\gamma^{\text {fcon3/fcon3 }}$ mutants (gray bars) compared with controls (white bars) at embryonic ages, but had recovered by P0, in contrast to Actin-Cre; Pcdh- $\gamma^{\text {fcon3/fcon3 }}$ mutants (black bars). Graphs represent means \pm SEM from 18 fields from 3 animals per genotype per time point. ${ }^{*} p<0.05 ;{ }^{* *} p<0.01 ;{ }^{* * *} p<0.001$.

\section{Discussion}

The close apposition of astrocyte processes and synaptic terminals in vivo and in vitro (Ventura and Harris, 1999; Cholet et al., 2002; Haber et al., 2006; Halassa et al., 2007) predicted that, in addition to releasing multiple synaptogenic factors (Mauch et al., 2001; Ullian et al., 2001; Christopherson et al., 2005), astrocytes would contribute to synaptogenesis via contact-dependent mechanisms. Consistent with this, Hama et al. (2004) demonstrated that neuronal integrins interact with astrocytes, resulting in PKC activation and a global increase of autapses in microisland cultures in vitro. It is not known, however, whether such a mechanism operates in vivo, nor has the astrocytic ligand for neuronal integrins been identified. Astrocytic ephrin-A3 is likely to mediate the effect of EphA4 signaling on hippocampal dendritic spine shape (Murai et al., 2003), although it is unclear whether such an interaction has an effect on synapse formation per se. Here, we have identified the 22-member $\gamma$-Pcdh family as the first known mediators of contact-dependent mechanisms through which perisynaptic astrocyte processes promote synaptogenesis both in vitro and in vivo.

Our analyses of cell-type-specific mutant cultures and animals suggest that there are two developmentally separable cellular mechanisms through which the $\gamma$-Pcdhs contribute to the control of synaptogenesis. Synaptogenesis was significantly reduced in developing astro-null cultures (Fig. 4) and in the spinal cords of embryonic astrocyte-restricted mutants in vivo (Fig. 7). Both in vivo and in vitro, synapse density eventually recovered toward wild-type levels with further maturation, but only if neurons expressed the $\gamma$-Pcdhs. This indicates that in immature neurons and/or at early stages of synapse formation and stabilization (which is likely asynchronous within a given population), astrocyte-neuron $\gamma$-Pcdh contacts are particularly critical, whereas, as neurons and/or synapses mature, neuron-neuron $\gamma$-Pcdh contacts suffice. Interestingly, restricted mutation of the $P c d h-\gamma$ locus in astrocytes in vivo did not lead to the increased neuronal apoptosis observed when all or many neurons lack $\gamma$-Pcdh function (Wang et al., 2002b; Lefebvre et al., 2008; Prasad et al., 2008), suggesting that the $\gamma$-Pcdhs promote neuronal survival specifically through neuron-neuron contacts, or that the transient delay in synaptogenesis we observe is not prolonged enough to result in apoptosis. A developmentally restricted requirement for astrocytic $\gamma$-Pcdh contacts in young neurons is consistent with a recent study (Barker et al., 2008) demonstrating that embryonic, but not postnatal, retinal ganglion cells require astrocyte contacts to become competent to receive synapses in vitro. Astrocyteneuron contacts may control synaptic receptivity by inducing changes in signaling pathways such as PKC (Hama et al., 2004), or the localization of cell-surface molecules such as neurexins (Barker et al., 2008), although the available in vitro data cannot distinguish between global effects and those mediated specifically at perisynaptic sites. It should be noted that perisynaptic contacts such as those mediated by the $\gamma$-Pcdhs could additionally enhance the effect of secreted factors, such as thrombospondins (Christopherson et al., 2005), by increasing their local concentration in three-dimensional tissues in vivo.

All $\gamma$-Pcdhs tested thus far can interact homophilically when expressed in cell lines (Sano et al., 1993; Obata et al., 1995; Frank et al., 2005; Fernández-Monreal et al., 2009) and our results are consistent with such a mechanism. In Cre-ER; Pcdh- $\gamma^{\text {fcon } 3 /+}$ mice, neuronal $\gamma$-Pcdhs can be observed at or near synaptic sites directly adjacent to astrocytic $\gamma$-Pcdhs (Fig. $2 D, E$ ). Furthermore, overall reductions in synapse density at 6 DIV were quantitatively similar in astro-null and neuron-null cocultures (Fig. 4), as well as in cocultures in which both astrocytes and neurons were mutant (data not shown) (see Weiner et al., 2005). Although models involving $\gamma$-Pcdh interaction with heterophilic ligands could conceivably explain this result, the most parsimonious explanation is that astrocytic $\gamma$-Pcdhs interact with neuronal $\gamma$-Pcdhs. Of course, even if interaction of $\gamma$-Pcdh family members is broadly homophilic, there could still be differential and/or combinatorial interaction between the 22 individual $\gamma$-Pcdh isoforms; determining such patterns, as well as characterizing the $\gamma$-Pcdh repertoire of individual astrocytes, will be an important future goal. Given the diversity of the adhesion molecules encoded by the $P c d h-\gamma$ gene cluster, our results raise the intriguing possibility that astrocyte-neuron contacts provide not only general support for synaptogenesis, but also molecular cues influencing the specificity of presynaptic and postsynaptic interactions. 


\section{References}

Barker AJ, Koch SM, Reed J, Barres BA, Ullian EM (2008) Developmental control of synaptic receptivity. J Neurosci 28:8150-8160.

Barres BA (2008) The mystery and magic of glia: a perspective on their roles in health and disease. Neuron 60:430-440.

Barry D, McDermott K (2005) Differentiation of radial glia from radial precursor cells and transformation into astrocytes in the developing rat spinal cord. Glia 50:187-197.

Bushong EA, Martone ME, Jones YZ, Ellisman MH (2002) Protoplasmic astrocytes in CA1 stratum radiatum occupy separate anatomical domains. J Neurosci 22:183-192.

Cholet N, Pellerin L, Magistretti PJ, Hamel E (2002) Similar perisynaptic glial localization for the na,$+ \mathrm{K}+-\mathrm{ATPase}$ alpha 2 subunit and the glutamate transporters GLAST and GLT-1 in the rat somatosensory cortex. Cereb Cortex 12:515-525.

Christopherson KS, Ullian EM, Stokes CC, Mullowney CE, Hell JW, Agah A, Lawler J, Mosher DF, Bornstein P, Barres BA (2005) Thrombospondins are astrocyte-secreted proteins that promote CNS synaptogenesis. Cell 120:421-433.

Colón-Ramos DA, Margeta MA, Shen K (2007) Glia promote local synaptogenesis through UNC-6 (netrin) signaling in C. elegans. Science 318:103-106

Derouiche A, Frotscher M (2001) Peripheral astrocyte processes: monitoring by selective immunostaining for the actin-binding ERM proteins. Glia 36:330-341.

Fernández-Monreal M, Kang S, Phillips GR (2009) Gamma-protocadherin homophilic interaction and intracellular trafficking is controlled by the cytoplasmic domain in neurons. Mol Cell Neurosci 40:344-353.

Frank M, Ebert M, Shan W, Phillips GR, Arndt K, Colman DR, Kemler R (2005) Differential expression of individual gamma-protocadherins during mouse brain development. Mol Cell Neurosci 29:603-616.

Guo C, Yang W, Lobe CG (2002) A cre recombinase transgene with mosaic, widespread tamoxifen-inducible action. Genesis 32:8-18.

Haber M, Zhou L, Murai KK (2006) Cooperative astrocyte and dendritic spine dynamics at hippocampal excitatory synapses. J Neurosci 26: 8881-8891.

Halassa MM, Fellin T, Takano H, Dong JH, Haydon PG (2007) Synaptic islands defined by the territory of a single astrocyte. J Neurosci 27:6473-6477.

Hama H, Hara C, Yamaguchi K, Miyawaki A (2004) PKC signaling mediates global enhancement of excitatory synaptogenesis in neurons triggered by local contact with astrocytes. Neuron 41:405-415.

Hayashi S, McMahon AP (2002) Efficient recombination in diverse tissues by a tamoxifen-inducible form of cre: a tool for temporally regulated gene activation/inactivation in the mouse. Dev Biol 244:305-318.

Lee J, Wu Y, Qi Y, Xue H, Liu Y, Scheel D, German M, Qiu M, Guillemot F, Rao M, Gradwohl G (2003) Neurogenin3 participates in gliogenesis in the developing vertebrate spinal cord. Dev Biol 253:84-98.

Lefebvre JL, Zhang Y, Meister M, Wang X, Sanes JR (2008) \{Gamma\}protocadherins regulate neuronal survival but are dispensable for circuit formation in retina. Development 135:4141-4151.

Lewandoski M, Meyers EN, Martin GR (1997) Analysis of Fgf8 gene function in vertebrate development. Cold Spring Harb Symp Quant Biol 62:159-168.

Mauch DH, Nägler K, Schumacher S, Göritz C, Müller EC, Otto A, Pfrieger FW (2001) CNS synaptogenesis promoted by glia-derived cholesterol. Science 294:1354-1357.

May MK, Biscoe TJ (1975) An investigation of the foetal rat spinal cord. I. Ultrastructural observations on the onset of synaptogenesis. Cell Tissue Res 158:241-249.

Murai KK, Nguyen LN, Irie F, Yamaguchi Y, Pasquale EB (2003) Control of hippocampal dendritic spine morphology through ephrin-A3/EphA4 sig naling. Nat Neurosci 6:153-160.

Novak A, Guo C, Yang W, Nagy A, Lobe CG (2000) Z/EG, a double reporter mouse line that expresses enhanced green fluorescent protein upon cremediated excision. Genesis 28:147-155.

Obata S, Sago H, Mori N, Rochelle JM, Seldin MF, Davidson M, St John T, Taketani S, Suzuki ST (1995) Protocadherin Pcdh2 shows properties similar to, but distinct from, those of classical cadherins. J Cell Sci 108:3765-3773.

Pfrieger FW, Barres BA (1997) Synaptic efficacy enhanced by glial cells in vitro. Science 277:1684-1687.

Phillips GR, Tanaka H, Frank M, Elste A, Fidler L, Benson DL, Colman DR (2003) Gamma-protocadherins are targeted to subsets of synapses and intracellular organelles in neurons. J Neurosci 23:5096-5104.

Prasad T, Wang X, Gray PA, Weiner JA (2008) A differential developmental pattern of spinal interneuron apoptosis during synaptogenesis: insights from genetic analyses of the protocadherin-\{gamma\} gene cluster. Development 135:4153-4164.

Rothstein JD, Martin L, Levey AI, Dykes-Hoberg M, Jin L, Wu D, Nash N, Kuncl RW (1994) Localization of neuronal and glial glutamate transporters. Neuron 13:713-725.

Sano K, Tanihara H, Heimark RL, Obata S, Davidson M, St John T, Taketani S, Suzuki S (1993) Protocadherins: a large family of cadherin-related molecules in central nervous system. EMBO J 12:2249-2256.

Stolt CC, Lommes P, Sock E, Chaboissier MC, Schedl A, Wegner M (2003) The Sox 9 transcription factor determines glial fate choice in the developing spinal cord. Genes Dev 17:1677-1689.

Tasic B, Nabholz CE, Baldwin KK, Kim Y, Rueckert EH, Ribich SA, Cramer P, Wu Q, Axel R, Maniatis T (2002) Promoter choice determines splice site selection in protocadherin alpha and gamma pre-mRNA splicing. Mol Cell 10:21-33.

Theodosis DT, Poulain DA, Oliet SH (2008) Activity-dependent structural and functional plasticity of astrocyte-neuron interactions. Physiol Rev 88:983-1008.

Ullian EM, Sapperstein SK, Christopherson KS, Barres BA (2001) Control of synapse number by glia. Science 291:657-661.

Vaughn JE (1989) Fine structure of synaptogenesis in the vertebrate central nervous system. Synapse 3:255-285.

Ventura R, Harris KM (1999) Three-dimensional relationships between hippocampal synapses and astrocytes. J Neurosci 19:6897-6906.

Wang X, Su H, Bradley A (2002a) Molecular mechanisms governing pcdhgamma gene expression: evidence for a multiple promoter and cisalternative splicing model. Genes Dev 16:1890-1905.

Wang X, Weiner JA, Levi S, Craig AM, Bradley A, Sanes JR (2002b) Gamma protocadherins are required for survival of spinal interneurons. Neuron 36:843-854.

Weiner JA (2006) Protocadherins and synapse development. In: Molecular mechanisms of synaptogenesis (Dityatev A, El-Husseini A, eds), New York: Springer.

Weiner JA, Wang X, Tapia JC, Sanes JR (2005) Gamma protocadherins are required for synaptic development in the spinal cord. Proc Natl Acad Sci U S A 102:8-14.

Wu Q, Maniatis T (1999) A striking organization of a large family of human neural cadherin-like cell adhesion genes. Cell 97:779-790.

Yamagata M, Herman JP, Sanes JR (1995) Lamina-specific expression of adhesion molecules in developing chick optic tectum. J Neurosci 15:4556-4571.

Zhuo L, Theis M, Alvarez-Maya I, Brenner M, Willecke K, Messing A (2001) hGFAP-cre transgenic mice for manipulation of glial and neuronal function in vivo. Genesis 31:85-94. 